\title{
THE SIGN OF LOMMEL'S FUNCTION
}

\author{
BY \\ J. STEINIG
}

\begin{abstract}
Lommel's function $s_{\mu, \nu}(x)$ is a particular solution of the differential equation $x^{2} y^{\prime \prime}+x y^{\prime}+\left(x^{2}-\nu^{2}\right) y=x^{\mu+1}$. It is shown here that $s_{\mu, \nu}(x)>0$ for $x>0$, if $\mu=\frac{1}{2}$ and $|\nu|<\frac{1}{2}$, or if $\mu>\frac{1}{2}$ and $|\nu| \leqq \mu$. This includes earlier results of R. G. Cooke's. The sign of $s_{\mu, \nu}(x)$ for other values of $\mu$ and $\nu$ is also discussed.
\end{abstract}

1. Introduction. In 1876, Lommel [6] considered the inhomogeneous differential equation

$$
z^{2} y^{\prime \prime}+z y^{\prime}+\left(z^{2}-v^{2}\right) y=z^{\mu+1},
$$

where $\mu$ and $\nu$ are complex parameters. He obtained two particular solutions: the Lommel functions of the first kind, $s_{\mu, v}(z)$, and of the second kind, $S_{\mu, v}(z)$. The homogeneous equation associated with (1) is

$$
z^{2} y^{\prime \prime}+z y^{\prime}+\left(z^{2}-\nu^{2}\right) y=0
$$

Bessel's equation.

The function $s_{\mu, \nu}(z)$ is defined for all pairs $\mu, \nu$ such that neither $\mu-\nu$ nor $\mu+\nu$ is an odd negative integer, and for all $z$ with $-\pi<\arg z \leqq \pi$, by the series

$$
s_{\mu, v}(z)=\frac{1}{4} z^{\mu+1} \sum_{n=0}^{\infty} \frac{(-1)^{n}\left(\frac{1}{2} z\right)^{2 n} \Gamma((\mu-\nu+1) / 2) \Gamma((\mu+\nu+1) / 2)}{\Gamma((\mu-\nu+n+3) / 2) \Gamma((\mu+\nu+n+3) / 2)} .
$$

The symmetry property

$$
s_{\mu, v}(z)=s_{\mu,-v}(z)
$$

is obvious from (3).

We shall consider $s_{\mu, v}(z)$ for $\mu$ and $\nu$ real, and positive $z$. Its importance arises from the formula $[1, \S 3.20],[9, \S 10.74]$

$$
\int x^{\mu} C_{v}(x) d x=x\left[C_{v}(x) s_{\mu, v}^{\prime}(x)-s_{\mu, v}(x) C_{v}^{\prime}(x)\right],
$$

in which $C_{v}(x)$ denotes any real solution of equation (2), that is,

$$
C_{v}(x)=\alpha J_{v}(x)+\beta Y_{v}(x),
$$

where $\nu, \alpha$ and $\beta$ are real, $x>0$, and $J_{v}(x)$ and $Y_{v}(x)$ denote the usual Bessel functions.

Presented to the Society, November 28, 1970; received by the editors December 22, 1970.

AMS 1970 subject classifications. Primary 33A70, 33A40; Secondary 26A33, 34C10, 44A20.

Key words and phrases. Lommel functions, Bessel functions, changes of sign, oscillation theorems, inhomogeneous Bessel equation.

Copyright (C) 1972, American Mathematical Society 
A case of particular interest is that in which $\mu=\nu$. Then, unless $\nu$ is half of an odd negative integer, we have

$$
s_{v, v}(x)=2^{v-1} \Gamma\left(\frac{1}{2}\right) \Gamma\left(\nu+\frac{1}{2}\right) H_{v}(x),
$$

where $\boldsymbol{H}_{v}(x)$ is Struve's function of order $\nu$ [1, (3.127)]. Now it is known $[9, \S 10.45]$ that $\boldsymbol{H}_{v}(x)>0$ for all $x>0$ if $\nu>\frac{1}{2}$, that $H_{1 / 2}(x)=(2 / \pi x)^{1 / 2}(1-\cos x)$, and that $\boldsymbol{H}_{\nu}(x)$ has an infinity of changes of sign if $\nu<\frac{1}{2}$. The corresponding problem for $s_{\mu, \nu}(x)$ is more difficult. R. G. Cooke [4] found conditions on $\mu$ and $\nu$ sufficient to ensure that $s_{\mu, v}(x)>0$ for all $x>0$. We may state his results as

THEOREM A. If $\nu \geqq 0$ and $\mu \geqq \nu+1$, then $s_{\mu, \nu}(x)>0$ for $x>0$. If $\nu \geqq \frac{1}{2}$ and $\mu \geqq \nu$, then $s_{\mu, v}(x)>0$ for $x>0$, unless $\mu=\nu=\frac{1}{2}$, when $s_{\mu, \nu}(x) \geqq 0$ for $x>0$.

The symmetry relation (4) gives the corresponding results for $\nu<0$.

Cooke's proof requires an expression for $s_{\mu, v}(x)$ as a fractional integral involving $J_{v}(x)$, and a previous result of his ([2] and [3]) which implies that if $\mu=0$ and $\nu>-1$, or $\mu=1-\nu$ and $\nu>\frac{1}{2}$, then

$$
\int_{0}^{\xi} t^{\mu} J_{v}(t) d t>0, \quad \xi>0 .
$$

We shall here consider the same problem. It will often be convenient to refer to a $(\mu, \nu)$-plane, and to associate with each pair $\mu, \nu$ the point with those coordinates.

We shall see that $s_{\mu, v}(x)>0$ for $x>0$ if $\mu \geqq \frac{1}{2}$ and $|\nu| \leqq \mu$, except when $\mu=|\nu|=\frac{1}{2}$, in which case $s_{\mu, v}(x) \geqq 0$. These inequalities define a larger region in the $(\mu, \nu)$-plane than Cooke's. If $\mu<\frac{1}{2}$, or if $\mu=\frac{1}{2}$ and $|\nu|>\frac{1}{2}$, then $s_{\mu, \nu}(x)$ changes sign infinitely often on $(0, \infty)$. If $\mu>\frac{1}{2}$ and $\mu<|\nu|-1, s_{\mu, v}(x)$ has an odd number of changes of sign on $(0, \infty)$. Finally, if $\mu>\frac{1}{2}$ and $|\nu|-1<\mu<|\nu|, s_{\mu, v}(x)$ has an even number of changes of sign on $(0, \infty)$; but I have not been able to decide whether this number is always positive. I shall show, however, that there are points $(\mu, \nu)$ in this region such that the corresponding $s_{\mu, v}(x)$ has changes of sign (an arbitrarily large number of them, in fact).

Our main tool is an oscillation theorem of Makai's [7] for second order differential equations. With it, we can determine the sign of the function

$$
h(\xi)=\int_{0}^{\xi} t^{\mu} C_{v}(t) d t, \quad \xi>0
$$

for certain pairs $\mu, \nu$. Then, by using (5), we can deduce results on the sign of $s_{\mu, v}(x)$.

2. Oscillation theorems. Our starting point is

THEOREM B. Let $y=y(x)$ be a solution of the differential equation $y^{\prime \prime}+\varphi(x) y=0$, where $\varphi$ is continuous and increasing on $\left(x_{0}, x_{2}\right)$. Let $x_{1}$ be the only zero of $y(x)$ on $\left(x_{0}, x_{2}\right)$. Further, assume that

$$
\lim _{x \rightarrow x_{0}+0} y(x)=\lim _{x \rightarrow x_{2}-0} y(x)=0
$$


Then,

$$
\int_{x_{0}}^{x_{1}}|y(x)| d x \geqq \int_{x_{1}}^{x_{2}}|y(x)| d x,
$$

with strict inequality if $\varphi$ is strictly increasing.

If $\varphi$ is decreasing on $\left(x_{0}, x_{2}\right)$, then $(8)$ is reversed.

Theorem $\mathrm{B}$ is due essentially to E. Makai [7], [8, §1.82], but the endpoint conditions (7) have been introduced to avoid difficulties which may arise when $\varphi$ is discontinuous at $x_{0}$, or at $x_{2}$. The monotonicity condition on $\varphi$ could be relaxed somewhat $[7, \S 2(d)]$, but we will not need this here.

From Theorem B we deduce

THEOREM 1. Let $y=y(x)$ be a solution of the differential equation

$$
\left(r(x) y^{\prime}\right)^{\prime}+p(x) y=0,
$$

where $r$ and $p$ are continuous, $p r$ is increasing and $r$ is positive, on $\left(x_{0}, x_{2}\right)$. Assume further that $\int_{x_{0}}^{x}(r(u))^{-1} d u$ converges. Let

$$
\lim _{x \rightarrow x_{0}+0} y(x)=\lim _{x \rightarrow x_{2}-0} y(x)=0,
$$

and let $x_{1}$ be the only zero of $y$ on $\left(x_{0}, x_{2}\right)$. Then,

$$
\int_{x_{0}}^{x_{1}} \frac{|y(x)|}{r(x)} d x \geqq \int_{x_{1}}^{x_{2}} \frac{|y(x)|}{r(x)} d x,
$$

with strict inequality if pr is strictly increasing. And if pr is decreasing (11) is reversed.

Proof. We transform the independent variable in (9) by setting

$$
t=f(x)=\int_{x_{0}}^{x} \frac{d u}{r(u)} .
$$

Equation (9) can then be written as

$$
d^{2} y / d t^{2}+r(g(t)) p(g(t)) y=0,
$$

where $g$ denotes the inverse of the (increasing) function $f[5, \mathrm{p} .235]$. Inequality (11) now follows by applying Theorem B to (12), and then changing the independent variable back to $x$.

In the sequel, we shall consider the equation

$$
\left(x^{1-2 \mu} y^{\prime}\right)^{\prime}+x^{-2 \mu-1}\left(x^{2}+\mu^{2}-\nu^{2}\right) y=0,
$$

which is of the form (9), with

$$
(p(x) r(x))^{\prime}=2 x^{-4 \mu-1}\left[(1-2 \mu) x^{2}+2 \mu\left(\nu^{2}-\mu^{2}\right)\right] .
$$

The integrand in (5), $x^{\mu} C_{v}(x)$, is a solution of equation (13) $[9, \S 4.31,(19)-(20)]$. We shall often choose $\mu=\frac{1}{2}$; (13) then becomes

$$
y^{\prime \prime}+\left(1+\left(1-4 \nu^{2}\right) / 4 x^{2}\right) y=0 .
$$


3. Lommel functions. The two Lommel functions $s_{\mu, v}(x)$ and $S_{\mu, v}(x)$ are related by the identity

$$
\begin{aligned}
s_{\mu, \nu}(x)=S_{\mu, \nu}(x)+ & 2^{\mu-1} \Gamma\left(\frac{\mu-\nu+1}{2}\right) \Gamma\left(\frac{\mu+\nu+1}{2}\right) \\
\cdot & {\left[\cos \frac{(\mu-\nu) \pi}{2} Y_{\nu}(x)-\sin \frac{(\mu-\nu) \pi}{2} J_{v}(x)\right], }
\end{aligned}
$$

whenever $s_{u, v}(x)$ is defined $[9, \S 10.71]$.

Now it can be shown $[9, \S 10.75]$ that $S_{\mu, v}(x) \sim x^{\mu-1}$, as $x \rightarrow+\infty$. On combining this with the relations

$$
J_{v}(x)=(2 / \pi x)^{1 / 2}\left[\cos \left(x-\frac{1}{2} v \pi-\frac{1}{4} \pi\right)+O(1 / x)\right]
$$

and

$$
Y_{v}(x)=(2 / \pi x)^{1 / 2}\left[\sin \left(x-\frac{1}{2} \nu \pi-\frac{1}{4} \pi\right)+O(1 / x)\right]
$$

$[9, \S 7.22]$, we see that as $x \rightarrow+\infty$, the dominant term on the right-hand side of (16) is

$$
2^{\mu-1}\left(\frac{2}{\pi x}\right)^{1 / 2} \Gamma\left(\frac{\mu-\nu+1}{2}\right) \Gamma\left(\frac{\mu+\nu+1}{2}\right) \sin \left(x-\frac{1}{2} \mu \pi-\frac{1}{4} \pi\right) \quad \text { or } \quad x^{\mu-1},
$$

according as $\mu<\frac{1}{2}$ or $\mu>\frac{1}{2}$. It follows that $s_{\mu, v}(x)$ has an infinity of changes of sign if $\mu<\frac{1}{2}$, but is positive for all sufficiently large $x$, if $\mu>\frac{1}{2}$. We now proceed to make this simple observation more precise. We begin by establishing

THEOREM 2. If $\mu<\frac{1}{2}$, or if $\mu=\frac{1}{2}$ and $|\nu|>\frac{1}{2}$, then $s_{\mu, \nu}(x)$ has an infinity of changes of sign on $(0, \infty)$.

Proof. For $\mu<\frac{1}{2}$ and $\nu$ unrestricted, the result follows as above. For $\mu=\frac{1}{2}$ and $|\nu|>\frac{1}{2}$, we apply Theorem B to the particular solution $y(x)=x^{1 / 2} J_{\nu}(x)$ of equation (15). It is clear from (14) that $p r$ is increasing on $(0, \infty)$ for such pairs $\mu, \nu$. Now let $j_{v, k}$ denote the $k$ th positive zero of $J_{v}(x)$. In (7), we may take $x_{0}=j_{v, k}$, for any $k$. And we may also take $x_{0}=0$, since $J_{v}(x)=O\left(x^{\nu}\right)$ as $x \rightarrow 0+$. Since $J_{v}(x)>0$ for $0<x<j_{v, 1}$ if $\nu>-1$, it follows from (8) that

$$
\int_{0}^{\xi} x^{1 / 2} J_{v}(x) d x>0, \quad \xi>0, \nu>\frac{1}{2} .
$$

Together with (5), this shows that $J_{v}(\xi) s_{1 / 2, v}^{\prime}(\xi)-s_{1 / 2, v}(\xi) J_{v}^{\prime}(\xi)>0$, for the righthand side of (5) vanishes at $x=0$ if $\beta=0$ and $\mu+\nu+1>0$, by (3). In particular,

$$
s_{1 / 2, v}\left(j_{v, k}\right) J_{v}^{\prime}\left(j_{v, k}\right)<0, \quad k=1,2, \ldots, \nu>\frac{1}{2} .
$$

But sgn $J_{v}^{\prime}\left(j_{v, k}\right)=(-1)^{k}$ for $\nu \geqq 0$; thus $s_{1 / 2, \nu}(x)$ must have an odd number of changes of sign between consecutive positive zeros of $J_{v}(x)$, if $\nu>\frac{1}{2}$. Because of (4), this is also true if $\nu<-\frac{1}{2}$, and the proof is complete.

Next, we turn to the case $\mu \geqq \frac{1}{2}$, and prove 
THEOREM 3. If $\mu \geqq \frac{1}{2}$ and $|\nu| \leqq \mu$, then $s_{\mu, v}(x)>0$ for all $x>0$, except if $\mu=|\nu|=\frac{1}{2}$, when $s_{\mu, v}(x) \geqq 0$.

Proof. For $\mu=|\nu|=\frac{1}{2}$ we have, from (6),

$$
s_{1 / 2,1 / 2}(x)=s_{1 / 2,-1 / 2}(x)=x^{-1 / 2}(1-\cos x) .
$$

For $\mu=\frac{1}{2}$ and $|\nu|<\frac{1}{2}$ we shall use Theorem B and (5). From (3), and familiar facts about the behavior of $J_{v}(x)$ and $Y_{v}(x)$ as $x \rightarrow 0+$, it is easily seen that we may take 0 as lower limit of integration in (5), and that the right-hand side of (5) vanishes at $x=0$, if $\mu>|\nu|-1$. Hence, for $\mu>|\nu|-1$ and $\xi>0$, we have

$$
\int_{0}^{\xi} x^{\mu} C_{v}(x) d x=\xi\left[C_{v}(\xi) s_{\mu, v}^{\prime}(\xi)-s_{\mu, v}(\xi) C_{v}^{\prime}(\xi)\right] .
$$

The particular choice $\xi=c_{v, k}$, the $k$ th positive zero of $C_{v}(x)$, yields

$$
\int_{0}^{c_{v, k}} x^{\mu} C_{\nu}(x) d x=-c_{v, k} s_{\mu, \nu}\left(c_{v, k}\right) C_{\nu}^{\prime}\left(c_{v, k}\right)
$$

Now $y(x)=x^{\mu} C_{v}(x)$ is a solution of (13). Since $\lim _{x \rightarrow 0+} x^{\mu} C_{v}(x)=0$ if $\mu>|v|,(10)$ is satisfied by $x_{0}=0$. Moreover, it is clear from (14) that $p r$ is strictly decreasing on $(0, \infty)$, if $\mu=\frac{1}{2}$ and $|\nu|<\frac{1}{2}$.

Thus, the hypotheses of Theorem B are satisfied by $y(x)=x^{1 / 2} C_{v}(x)$ on each of the intervals $\left(0, c_{v, 2}\right)$ and $\left(c_{v, k}, c_{v, k+2}\right), k \geqq 1$, if $|\nu|<\frac{1}{2}$. It follows that

$$
\left\{\int_{0}^{c_{v, k}} x^{1 / 2} C_{\nu}(x) d x\right\}\left\{\int_{0}^{c_{v, k+1}} x^{1 / 2} C_{\nu}(x) d x\right\}<0, \quad k \geqq 1,|\nu|<\frac{1}{2} .
$$

Since $C_{v}^{\prime}\left(c_{v, k}\right) C_{v}^{\prime}\left(c_{v, k+1}\right)<0[9, \S 15.21]$, it follows from (18) and (19) that

$$
s_{1 / 2, \nu}\left(c_{v, k}\right)>0, \quad k \geqq 1,|\nu|<\frac{1}{2} .
$$

But $x^{1 / 2} C_{v}(x)$ is an arbitrary nonnull solution of (15). Therefore, (20) implies that $s_{1 / 2, v}(x)>0$ for all $x>0$ if $|\nu|<\frac{1}{2}$, since any $x>0$ is a zero of some nonnull solution of (15). Together with (17), this proves Theorem 3 for $\mu=\frac{1}{2}$. And if we use the integrals

$$
\begin{aligned}
& s_{\mu+\sigma, v+\sigma}(x)=2 x^{\sigma} \frac{\Gamma((\mu+\nu+2 \sigma+1) / 2)}{\Gamma(\sigma) \Gamma((\mu+\nu+1) / 2)} \int_{0}^{\pi / 2} s_{\mu, v}(x \sin \theta) \sin ^{\nu+1} \theta \cos ^{2 \sigma-1} \theta d \theta, \\
& s_{\mu+\sigma, v-\sigma}(x)=2 x^{\sigma} \frac{\Gamma((\mu+\nu+2 \sigma+1) / 2)}{\Gamma(\sigma) \Gamma((\mu-\nu+1) / 2)} \int_{0}^{\pi / 2} s_{\mu, v}(x \sin \theta) \sin ^{1-\nu} \theta \cos ^{2 \sigma-1} \theta d \theta,
\end{aligned}
$$

both valid for $\sigma>0$ and $\mu \pm \nu>-1[1, \S 3.20]$, the theorem's truth for $\mu>\frac{1}{2}$ is an immediate consequence of its truth for $\mu=\frac{1}{2}$.

An intermediate situation between those of the last two theorems is described by

THEOREM 4. Let $\mu>\frac{1}{2}$. If $\mu<|\nu|-1$, then $s_{\mu, v}(x)$ has an odd number of changes of sign on $(0, \infty)$. If $|\nu|-1<\mu<|\nu|$, then $s_{\mu, \nu}(x)$ has an even number of changes of sign (perhaps none) on $(0, \infty)$. 
Proof. From (3) we have $s_{\mu, v}(x) \sim x^{\mu+1} /\left((\mu+1)^{2}-v^{2}\right)$, as $x \rightarrow 0+$. On the other hand, it follows from our discussion of (16) that when $\mu>\frac{1}{2}, s_{\mu, \nu}(x) \sim x^{\mu-1}$, as $x \rightarrow+\infty$. The conclusion is now obvious.

I have not been able to decide whether the even number alluded to in Theorem 4 is in fact always positive. However, I can show that if the point $(\mu, \nu)$, with $\mu>\frac{1}{2}$ and $|\nu|-1<\mu<|\nu|$, is close enough to the line $\mu=\frac{1}{2}$, or to one of the lines $\mu=|\nu|-1$, then the corresponding $s_{\mu, v}(x)$ changes sign. In fact, by choosing the point $(\mu, \nu)$ close enough to the line $\mu=\frac{1}{2}$ in the region $\mu>\frac{1}{2},|\nu|-1<\mu<|\nu|$, we can find Lommel functions with an arbitrarily large number of changes of sign.

This last statement can be verified as follows. We may assume $\nu>0$. By applying Theorem 1 to (13), we see that if

$$
(1-2 \mu) x^{2}+2 \mu\left(\nu^{2}-\mu^{2}\right)>0 \text { for } 0<x<\bar{x},
$$

and if $j$ is the largest zero of $J_{v}(x)$ in $(0, \bar{x}]$, then

$$
\int_{0}^{x} t^{3 \mu-1} J_{v}(t) d t>0 \text { for } 0<x \leqq j,
$$

since (10) is satisfied by $y(x)=x^{\mu} J_{v}(x)$ and $x_{0}=0$, if $\mu+\nu>0$. For $\mu \geqq \frac{1}{2}$, this implies that

$$
\int_{0}^{x} t^{u} J_{v}(t) d t>0 \text { for } 0<x \leqq j
$$

by the second mean value theorem.

Thus, if $(0, \bar{x}]$ contains at least two positive zeros of $J_{v}(t)$, then (22) holds with $j=j_{v, 2}$, and hence also with $j=j_{v, 3}$. As before, if $\mu>\nu-1$, this will imply that $s_{\mu, v}(x)$ changes sign on $\left(j_{v, 1}, j_{v, 2}\right)$ and on $\left(j_{v, 2}, j_{v, 3}\right)$. Now for $\mu>\frac{1}{2}$, (21) holds if and only if $\mu<|\nu|$ and $|\bar{x}| \leqq\left(2 \mu\left(\nu^{2}-\mu^{2}\right) /(2 \mu-1)\right)^{1 / 2}$. Therefore, $s_{\mu, v}(x)$ will change sign if $\mu>\frac{1}{2}, \nu-1<\mu<\nu$, and

$$
j_{v, 2} \leqq\left(2 \mu\left(v^{2}-\mu^{2}\right) /(2 \mu-1)\right)^{1 / 2} .
$$

Now if we choose some $\nu>\frac{1}{2}$, and keep it fixed, then the left-hand side of (23) is fixed. But the right-hand side tends to $+\infty$, as $\mu \rightarrow \frac{1}{2}+0$. Hence for each $\nu, \frac{1}{2}<\nu<\frac{3}{2}$, we can find a $\mu\left(\mu>\frac{1}{2}, \nu-1<\mu<\nu\right)$ such that $s_{\mu, \nu}(x)$ has at least two changes of sign. In order to produce a Lommel function with at least $2 n$ changes of sign, it suffices to satisfy the inequality obtained by writing $j_{v, 2 n}$ for $j_{v, 2}$ in (23).

We now turn our attention to points near the line $\mu=\nu-1$, in the region $\mu>\frac{1}{2}$, $\nu-1<\mu<\nu$. Here, we use (16). We fix $\nu \geqq \frac{3}{2}$, and choose $x^{*}>0$ such that $J_{v}\left(x^{*}\right)<0$. Then, we let $\mu \rightarrow(\nu-1)+0$. Now $S_{\mu, \nu}\left(x^{*}\right)$ tends to a limit, as $\mu \rightarrow \nu-1[9, \S 10.73]$. And

$$
\lim _{\mu \rightarrow \nu-1}\left[\cos \frac{(\mu-\nu) \pi}{2} Y_{v}\left(x^{*}\right)-\sin \frac{(\mu-\nu) \pi}{2} J_{v}\left(x^{*}\right)\right]=J_{\nu}\left(x^{*}\right)
$$


Hence, for $x=x^{*}$, the right-hand side of (16) tends to $-\infty$ as $\mu \rightarrow(\nu-1)+0$, because of the factor $\Gamma((\mu-\nu+1) / 2)$. Therefore, $s_{\mu, \nu}\left(x^{*}\right)<0$ for an appropriate choice of $\mu$.

\section{REFERENCES}

1. A. W. Babister, Transcendental functions satisfying nonhomogeneous linear differential equations, Macmillan, New York, 1967. MR 34 \#6158.

2. R. G. Cooke, Gibbs's phenomenon in Fourier-Bessel series and integrals, Proc. London Math. Soc. (2) 27 (1928), 171-192.

3. - A monotonic property of Bessel functions, J. London Math. Soc. 12 (1937), 180-185.

4. - On the sign of Lommel's function, J. London Math. Soc. 7 (1932), 281-283.

5. W. Leighton, Ordinary differential equations, 3rd ed., Wadsworth, Belmont, Calif., 1970.

6. E. Lommel, Ueber eine mit den Besselschen Functionen verwandte Function, Math. Ann. 9 (1876), 425-444.

7. E. Makai, On a monotonic property of certain Sturm-Liouville functions, Acta Math. Acad. Sci. Hungar. 3 (1952), 165-172. MR 14, 872.

8. G. Szegö, Orthogonal polynomials, Amer. Math. Soc. Colloq. Publ., vol. 23, Amer. Math. Soc., Providence, R. I., 1959. MR 21 \#5029.

9. G. N. Watson, A treatise on the theory of Bessel functions, 2nd ed., Cambridge Univ. Press, Cambridge; Macmillan, New York, 1944. MR 6, 64.

Department of Mathematics, University of Illinois, Urbana, Illinois 61801

Current address: Institut Mathématique, Université de Genève, Genève, Switzerland 